Supporting Information

\title{
Microplasma-Enabled Surfaced-Functionalized Silicon Quantum Dots for Label-Free Detection of Dopamine
}

Gui-Yi Chang, Darwin Kurniawan, Yi-Ju Chang, and Wei-Hung Chiang*

Department of Chemical Engineering, National Taiwan University of Science and Technology, Taipei 10617, Taiwan

* Emails of corresponding authors: whchiang@mail.ntust.edu.tw(W.-H. C.) 


\section{S1. Materials}

[3-(2-Aminoethylamino)propyl]trimethoxysilane (AEAPTMS) ( $\geq 96 \%$, CAS: 1760-24-3) and glutamic acid (Glu, 99\%, CAS: 56-86-0) was obtained from Alfa Aesar. Cysteine (Cys, 98+\%, CAS: 52-90-4), maltose (Mat, 92+\%, CAS: 6363-53-7), sucrose (Sur, 99+\%, CAS: 57-50-1), and phenethylamine (CAS: 64-04-0) were purchased from ACROS Organics. Sodium hydroxide (NaOH, $\geq 98 \%$, CAS: 1310-73-2), dopamine hydrochloride (DA, CAS: 62-31-7), fructose (Fru, $\geq 99 \%$, CAS: 57-48-7), ascorbic acid (AA, CAS: 50-81-7), epinephrine (EP, CAS: 51-43-4), and tyramine (CAS: 51-67-2) were obtained from Sigma Aldrich Co., Ltd. Adenosine triphosphate (ATP, 98\%, CAS: 56-65-5) was purchased from AK Scientific, Inc. Hydrochloric acid (HCl, reagent grade, 37\%, CAS: 7647-01-0) was purchased from Scharlau. Pt foil $(99.95 \%, 20 \mathrm{~mm} \times 20 \mathrm{~mm} \times 0.1 \mathrm{~mm})$ was purchased from Guv Team International Co., Ltd. Taiwan. All chemicals were used without purification. Deionized (DI) water was used throughout the work.

\section{S2. Synthesis of SiQD}

SiQDs were synthesized in aqueous solutions using a direct current (dc) microplasma electrochemical reactor operated at ambient conditions, shown in Figure 1a. The experimental setup is similar our previous worls. $^{1-2}$ The reactor is consisted of a platinum foil $(1 \mathrm{~cm} \times 1 \mathrm{~cm})$ anode immersed in an electrolyte and a microplasma cathode. The distances between the anode and cathode and cathode to the liquid surface are approximately $3 \mathrm{~cm}$ and $1.5 \mathrm{~mm}$, respectively. A flow of $25 \mathrm{sccm}$ argon (Ar) was introduced in a hollow stainless steel capillary (i.d. $=180 \mu \mathrm{m})$ to facilitate the formation of a microplasma. A dc power supply was used to ignite and maintain a stable plasma with a constant current of $7 \mathrm{~mA}$. The process time was kept at $0.5 \mathrm{~h}$. The precursor solution was prepared by mixing AEAPTMS (0.2 mL), $0.1 \mathrm{M} \mathrm{NaOH}(2.5 \mathrm{~mL})$ and DI water $(9.8 \mathrm{~mL})$ 


\section{S3. Characterization}

Absorbance spectroscopy was carried out with a JASCO V676 absorbance spectrophotometer with a matched pair of $1 \mathrm{~cm}$ path length quartz cuvettes. PL spectroscopy was performed at $25^{\circ} \mathrm{C}$ on the liquid as-produced SiQD dispersion. Excitation and emission spectra were obtained by a commercial spectrometer (Horiba Jobin Yvon Nanolog-3 spectrofluorometer) equipped with an InGaAs NIR detector and a $20 \mathrm{~nm}$ bandpass for both emission and excitation. The PL spectra were scaled according to the measured excitation power. Micro Raman measurement was conducted at rat $25{ }^{\circ} \mathrm{C}$ with a JASCO 5100 spectrometer (laser excitation wavelength $\lambda=532 \mathrm{~nm}$ ). Thin films of SiQDs were deposited on Si wafers by drop-casting and dried in ambient conditions for $24 \mathrm{hr}$. The laser power was maintained at $0.1 \mathrm{~mW}$ to avoid heat generation by the laser. Silicon wafer was used to calibrate Raman shifts using the $520 \mathrm{~cm}^{-1}$ peak. FITR spectra was collected with a FTIR-iS10 equipped with attenuated total reflection (ATR) module within a range of 1000- $4000 \mathrm{~cm}^{-1}$. TEM was performed using a cold-field emission TEM (FEI Tecnai $^{\mathrm{TM}}$ G2 F-20 S-TWIN) with an accelerating voltage of $200 \mathrm{kV}$. TEM samples were prepared from solution dry-casting of SiQDs dispersion onto formvar/carbon-coated copper grids (300 mesh, EM Resolutions Ltd.). XPS (ULVAC-PHI, PHI Quantera SXM, Japen) was carried out witha monochromatic $\mathrm{Al} \mathrm{K} \alpha \mathrm{X}$-ray radiation $(10 \mathrm{kV}, 10 \mathrm{~mA})$. Samples were dropped on Nifoils and dried on a hotplate at $40^{\circ} \mathrm{C}$ in ambient condition.

\section{S4 Selectivity detection of biomarkers}

Each solution including dopamine (DA), ascorbic acid (AA), adenosine triphosphate (ATP), D-fructose

(Fru), sucrose (Sur), maltose (Mat), glutamic acid(Glu), and cysteine (Cys) $(2.5 \mathrm{~mL}, 100 \mu \mathrm{M})$ were mixed 
with SiQD solutions. After $1 \mathrm{~h}$ incubation time, the PL intensity of the mix dispersion was measured at $340 \mathrm{~nm}$ excitation wavelength.

\section{S5. Quantitative DA detection}

DA solutions with different concentrations $(2.5 \mathrm{ml})$ mixed with SiQDs solution $(0.5 \mathrm{ml})$. After $1 \mathrm{~h}$ incubation time, the PL intensity of the mix dispersion was measured at $340 \mathrm{~nm}$ excitation wavelength.

\section{S6. pH effect study}

The $1 \mathrm{M}$ phosphate buffer saline (PBS) solutions with different $\mathrm{pH}$ values were prepared by adjusting the $\mathrm{pH}$ values with $\mathrm{HCl}$ and $\mathrm{NaOH}$. Then DA solutions with different concentrations (2.2 ml) with mixed with SiQDs solution $(0.5 \mathrm{ml})$ and $\mathrm{pH}$-adjusted PBS solutions $(0.3 \mathrm{~mL})$. After $1 \mathrm{~h}$ incubation time, the PL intensity of the mix dispersion was measured at $340 \mathrm{~nm}$ excitation wavelength.
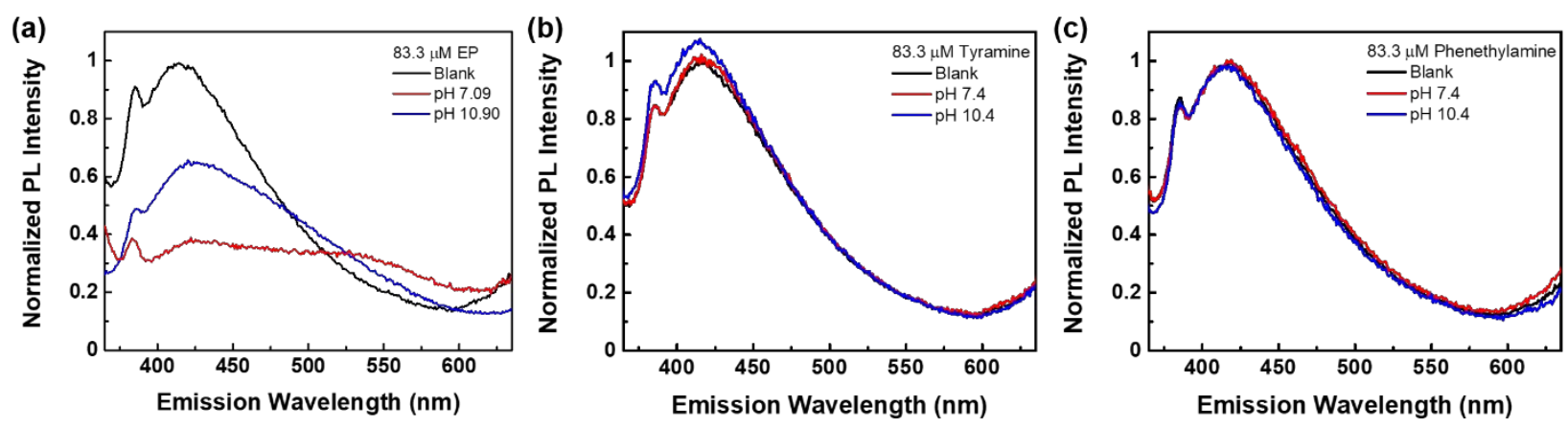

Figure S1. The PL spectra of SiQDs with the addition of $83.3 \mu \mathrm{M}$ of (a) epinephrine (EP), (b) tyramine, and (c) phenethylamine after 1 hour incubation under neutral (red color) and alkaline (blue color) $\mathrm{pH}$ at $25^{\circ} \mathrm{C}$. The blank PL spectrum of SiQDs is shown for comparison. 


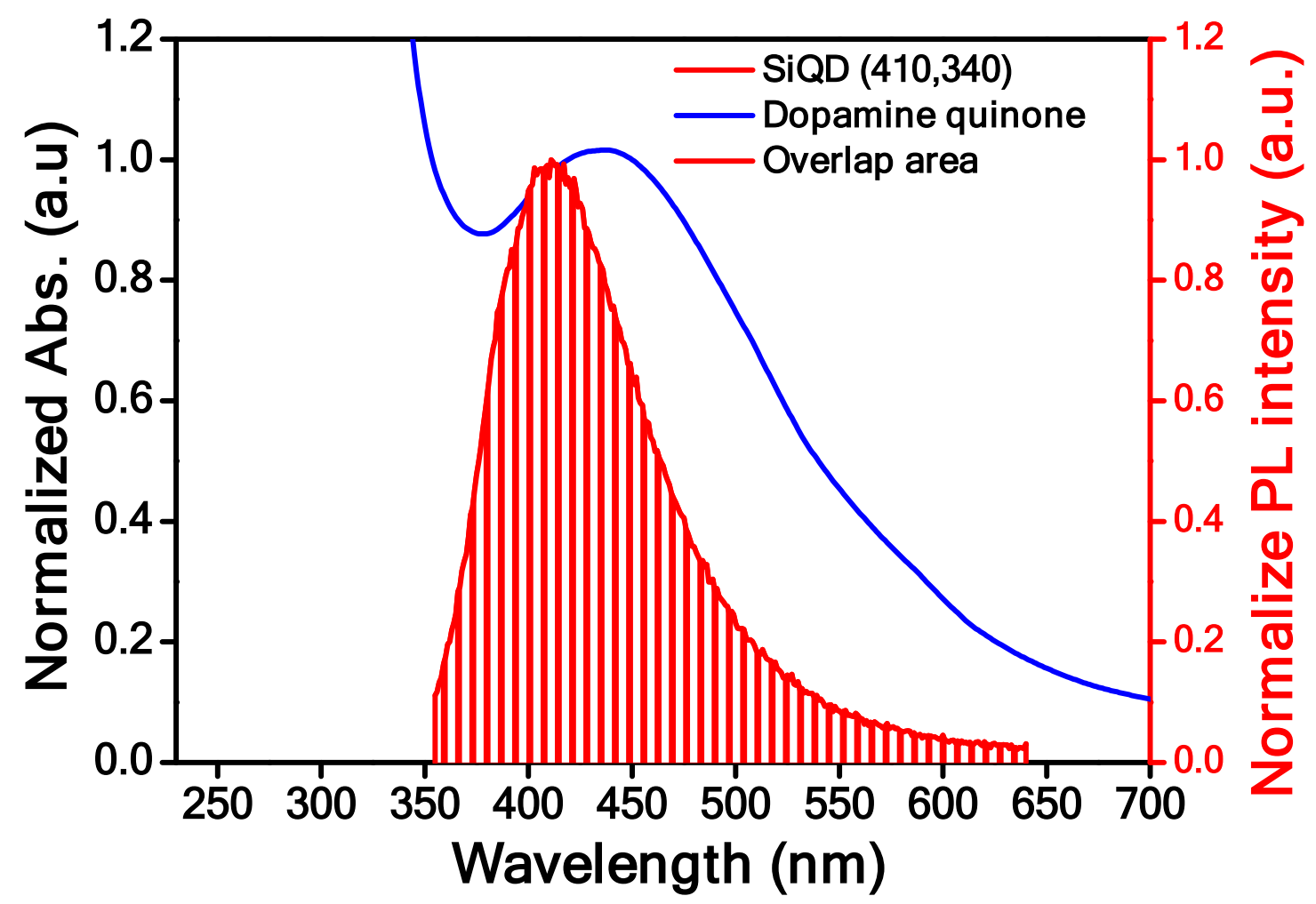

Figure S2. The overlapped area of PL emission spectrum of SiQDs and absorbance spectrum of DA-Q. 

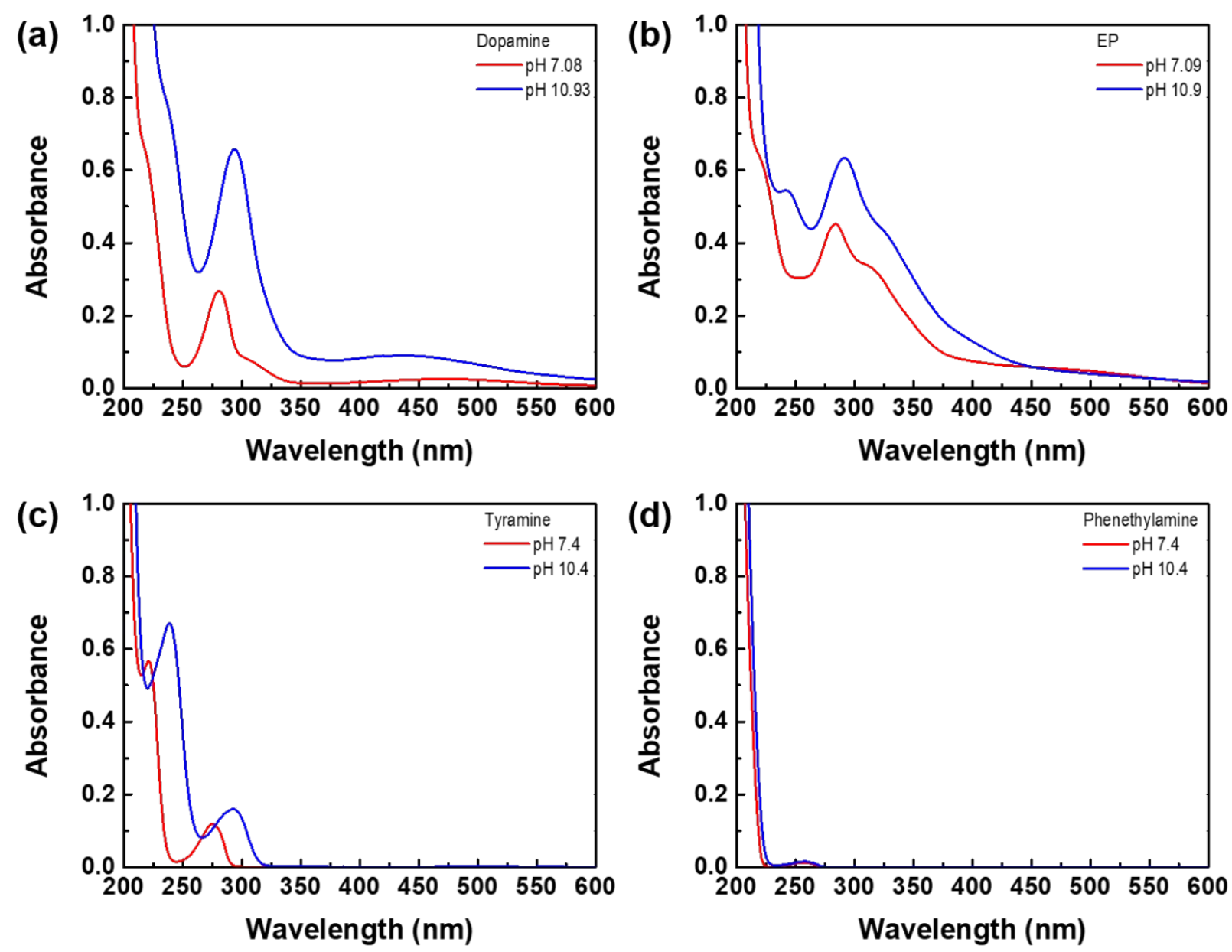

Figure S3. The absorption spectra of $83.3 \mu \mathrm{M}$ (a) dopamine (DA), (b) EP, (c) tyramine, and (d) phenethylamine under neutral (red color) and alkaline (blue color) $\mathrm{pH}$ at $25^{\circ} \mathrm{C}$.

\section{Reference}

1. Kurniawan, D.; Chiang, W.-H., Microplasma-Enabled Colloidal Nitrogen-Doped Graphene Quantum Dots for Broad-Range Fluorescent pH Sensors. Carbon 2020, 167, 675-684.

2. Yeh, P.-C.; Yoon, S.; Kurniawan, D.; Chung, Y. G.; Chiang, W.-H., Unraveling the Fluorescence Quenching of Colloidal Graphene Quantum Dots for Selective Metal Ion Detection. ACS Appl. Nano Mater. 2021, 4 (6), 5636-5642. 